\title{
High frequency of methicillin-resistant Staphylococcus aureus in Peshawar Region of Pakistan
}

\author{
Asad Ullah', Muhammad Qasim*, Hazir Rahman', Jafar Khan', Mohammad Haroon², Niaz Muhammad', \\ Abdullah Khan ${ }^{1}$ and Noor Muhammad ${ }^{3}$
}

\begin{abstract}
Staphylococcus aureus is an increasing problem in clinical practice because of reduced susceptibility to available antibiotics. The objective of the study was to determine the frequency of Methicillin-resistant S. aureus (MRSA) in Peshawar, Pakistan. Clinical isolates of $S$. aureus were subjected to determination of antibiotic resistance, MICs and inducible clindamycin resistance (ICR). Out of total $280 \mathrm{~S}$. aureus isolates, the frequency of MRSA was $36.1 \%(n=101)$. MRSA infection was found higher among the age group 50-59 years (60.71\%, OR 3.09), followed by 20-29 years (47.5\%, OR 1.74). Frequency of MRSA in female and male was 39.8 and $34 \%$ respectively. MRSA was more frequent in blood specimens (48.7\%, OR 2.14). The frequency of community and hospital acquired MRSA was 42 and $34.8 \%$ respectively. MRSA showed high resistance (100\%) to penicillin and cefoxitin followed by erythromycin ( $99 \%)$. While MRSA exhibited $100 \%$ susceptibility to vancomycin and linezolid. We have also found 7 vancomycin intermediate sensitive S. aureus (VISA) isolates. ICR was observed in $15.84 \%(n=16)$ of MRSA isolates. It is concluded that MRSA is potential threat to public health in Peshawar. Vancomycin and linezolid could be prescribed as a drug of choice in treating MRSA associated infections. In addition, ICR should be routinely checked to avoid clindamycin treatment failure.
\end{abstract}

Keywords: Epidemiology, Drug resistance, S. aureus, MRSA, Peshawar

\section{Background}

Staphylococcus aureus (S. aureus) is a serious risk to public health as it causes human infections ranges from wound abscess to life threatening conditions (Deleo et al. 2010). Emergence of antibiotics resistant S. aureus especially Methicillin-resistant Staphylococcus aureus (MRSA) is a worldwide problem in both healthcare and community settings (Chambers and DeLeo 2009). MRSA can be transmitted from person to person via skin or the sharing of contaminated objects. In addition, MRSA can evade host immune system and virulence factors disseminated render this bug with limited therapeutic options available (Holcomb et al. 2008). MRSA have capability to breed in the presence of methyl penicillin and its

\footnotetext{
*Correspondence: qasim89@gmail.com

1 Department of Microbiology, Kohat University of Science and Technology, Kohat, Khyber Pakhtunkhwa 26000, Pakistan Full list of author information is available at the end of the article
}

derivatives like methicillin. Resistance to methicillin is mediated by $m e c-A$ gene, which encodes the polypeptide PBP2a protein (Oliveira et al. 2006). The $m e c-A$ gene have also insertion sites for transposons and plasmids which assist resistance to other antibiotic groups. Accordingly, cross-resistance to non-beta-lactam antibiotic groups including quinolones, sulfamethoxazole, macrolides, aminoglycoside and lincomycin were frequently observed in MRSA isolates (Chambers 2001).

It has been reported that the MRSA infections are not limited to human but it can also infect domestic or farm animals such as horses, sheep, goat, and cattle (Saleha and Zunita 2010). MRSA has worldwide distribution but their frequency varies among various countries. The frequency of MRSA in Pakistan and India has been shown to be high as compared to northern Europe (Anwar et al. 2004). In Pakistan, the prevalence of methicillin resistance in $S$. aureus has been observed with range from 42 to $51 \%$ (Akinkunmi and Lamikanra 2012). With the 
increase of MRSA associated infections, the use of glycopeptides (vancomycin and teicoplanin) are also rising gradually. The irrational and indiscriminate use of glycopeptides results in the emergence of even low-level vancomycin resistance in S. aureus. Such resistance, though rare, but it is an emerging threat because of its potential to disseminate rapidly (Perwaiz et al. 2007). The present study was designed to investigate the frequency of MRSA in Peshawar. Findings of the study will be helpful for devising an appropriate hospital antibiotic stewardship policy to reduce the chances of $S$. aureus associated infections in the area.

\section{Methods}

\section{Ethical considerations}

The current study was approved by departmental research ethics committee (DREC) of the Department of Microbiology, Kohat University of Science and Technology, Kohat, Pakistan.

\section{Samples description}

The present study was carried out at Peshawar, the provincial capital of Khyber Pakhtunkhwa, Pakistan with confluence of local and Afghan refugee's population. Clinical samples received at Pathology Department of Rehman Medical and Teaching Institute Peshawar, Pakistan from September 2012 to September 2013 were included in the study. An informed written consent was taken from patients to collect data regarding patient's history, age and sex. In the present study different clinical samples were randomly collected from patients who visited or admitted in the hospital during study duration. MRSA Infection origin (hospital acquired vs community acquired) was also determined. Hospital acquired infection is an infection that develops in the hospital and was not present at the time of admission while a community-acquired infection has been defined as an infection that was present at the time of admission in the hospital. This definition is as per CDC definition (Garner et al. 1988; Salgado et al. 2003).

\section{Culture and biochemical identification of $S$. aureus}

Randomly collected clinical samples such as pus, fluid, blood, tissue and throat were processed for bacteria culturing followed by $S$. aureus identification based on Gram's staining, culture characteristics and biochemical reactions such as Catalase, Coagulase and DNase (Perwaiz et al. 2007). A total of 280 samples which were positive for $S$. aureus were further processed for antibiotic susceptibility to screen MRSA and MSSA.

\section{Phenotypic based detection of MRSA}

All confirmed S. aureus isolates were screened for methicillin resistance using cefoxitin (Fox) disc and oxacillin resistant screening agar base medium (ORSB) method as described (Velasco et al. 2005). Briefly, bacterial colony was picked up from overnight culture and inoculated into ORSAB medium followed by incubation at $35{ }^{\circ} \mathrm{C}$ for 24 to $48 \mathrm{~h}$. Presence of growth on ORSB was indicative of Oxacillin resistance. To check FOX resistance, bacterial lawn was prepared on Mueller-Hinton agar and FOX disk was placed on it, followed by incubation at $37^{\circ} \mathrm{C}$ for overnight. Growth around the FOX disk was indicative of FOX resistance.

\section{Molecular based detection of MRSA}

Total DNA was extracted using Nucleospin ${ }^{\circledR}$ tissue genomic DNA kit according to the vendor protocol (Macherey-Nagel, Germany). The purified DNA was subjected to PCR based amplification of mecA gene using specific primers (Forward: 5'-AAAATCGATGGT AAAGGTTGGC-3'; Reverse 5'-AGTTCTGCAGTACC GGATTTGC-3') as described earlier (Lee 2003). The amplified product was resolved on agarose gel electrophoresis and then visualized under UV transilluminator.

\section{Antibiotic susceptibility assay}

Antibiotic susceptibility test was carried out by disc diffusion method (Velasco et al. 2005). Bacterial overnight culture matched with 0.5 McFarland standards was inoculated on Mueller-Hinton agar plates and various antibiotic discs were placed followed by incubation at $37{ }^{\circ} \mathrm{C}$ for $24 \mathrm{~h}$. Afterward, an antibiotic zone of inhibition was measured and interpreted as per the CLSI guidelines (CLSI 2012).

\section{MIC determination of vancomycin against MRSA isolates by E-test method}

The MIC to vancomycin was performed by E-test method (Oxoid, England) and interpreted as per the CLSI guidelines (CLSI 2012).

\section{Detection of inducible clindamycin resistance by D-test} Inducible clindamycin resistance for MRSA isolates was checked by D-test as described earlier (Yilmaz et al. 2007). Briefly, bacterial culture at 0.5 McFarland equivalents was inoculated onto a MHA plate, followed by placement of erythromycin $(15 \mathrm{mg})$ and clindamycin $(2 \mathrm{mg})$ disks at $15 \mathrm{~mm}$ apart. Plate was then incubated at $35^{\circ} \mathrm{C}$ for overnight. A "D" shaped (blunted) clindamycin zone of inhibition towards erythromycin disc was interpreted as positive inducible clindamycin resistance. 


\section{Statistical analysis}

Numerical data was analyzed by Chi square test, odd ratio (OR) and $95 \%$ confidence interval to determine statistical significance in the age, gender, infection origin (i.e. community vs. hospital) and antibiotic susceptibility pattern for MRSA and MSSA.

\section{Results}

\section{Frequency of MRSA and MSSA}

Out of total $280 \mathrm{~S}$. aureus isolates, the frequency of MRSA and MSSA was $36.1 \%(\mathrm{n}=101)$ and $63.9 \%$ $(\mathrm{n}=179)$ respectively. Both phenotypic (Cefoxitin disc and ORSAB) and Molecular (mecA detection) based MRSA screening methods showed similar results.

Specimen wise distribution showed that MRSA was most frequent in blood (48.7 \%, OR 2.14), followed by fluids (41.7 \%, OR 1.27), and pus (36.7 \%, OR 1.04). MSSA was most frequent in throat specimens $(82.6 \%)$ followed by tissue samples ( $80 \%)$. (Table 1$)$.

The frequency of MRSA was found to be 39.8 and $34 \%$ for female and male respectively. On the other hand, the frequency of MSSA in male and female was 66.6 and $60 \%$ respectively (Table 1).
Methicillin-resistant $S$. aureus infection was higher among the age group $50-59$ years $(60.71 \%$, OR 3.09$)$, followed by $20-29$ years ( $47.5 \%$, OR 1.74$)$, and $1-9$ years (37.9\%, OR 1.10). MSSA infection was found higher among the age group 70-79 years (80\%), followed by 60-69 years (75\%) (Table 1$)$.

Among MRSA, the frequency of community acquired MRSA and hospital acquired MRSA was $42 \%(\mathrm{n}=21)$ and $34.8 \%(n=80)$ respectively. The frequency of community acquired MSSA and hospital acquired MSSA was 58 and $65.2 \%$ respectively (Table 1 ).

\section{Antibiotic susceptibility pattern of MRSA and MSSA}

The current study showed that all MRSA isolates were $100 \%$ resistant to penicillin and cefoxitin; however entirely (100 \%) susceptible to linezolid and vancomycin followed by rifampicin (81.2\%), chloramphenicol (77.2 \%), clindamycin (75.2\%), minocycline (67.3\%) and cotrimoxazole $(65.3 \%)$ (Table 2). The data was further investigated for multi-drugs resistance (MDR) MRSA and extended drugs resistant (XDR) MRSA as per CLSI (2012), which showed that $15.84 \%$ and $6.93 \%$ MRSA were observed to be MDR and XDR respectively. D-test

Table 1 Prevalence of MRSA and MSSA isolated from Peshawar region of Khyber Pakhtunkhwa, Pakistan

\begin{tabular}{|c|c|c|c|c|c|c|c|c|}
\hline \multirow[t]{2}{*}{ Variables } & \multirow[t]{2}{*}{ S. aureus (N) } & \multirow[t]{2}{*}{ MRSA n (\%) } & \multirow[t]{2}{*}{ MSSA n (\%) } & \multirow[t]{2}{*}{$x^{2}$} & \multirow[t]{2}{*}{ OR } & \multicolumn{2}{|c|}{$95 \%$ Confidence interval } & \multirow[t]{2}{*}{$p$ value } \\
\hline & & & & & & Lower limit & Upper limit & \\
\hline \multicolumn{9}{|l|}{ Age (years) } \\
\hline $1-9$ & 66 & $25(37.9)$ & $41(62.1)$ & 0.122 & 1.10 & 0.6257 & 1.9593 & 0.727 \\
\hline $10-19$ & 20 & $7(35)$ & $13(65)$ & 0.011 & 0.95 & 0.3666 & 2.4663 & 0.918 \\
\hline $20-29$ & 40 & $19(47.5)$ & $21(52.5)$ & 2.643 & 1.74 & 0.8872 & 3.425 & 0.104 \\
\hline $30-39$ & 34 & $11(32.3)$ & $23(67.7)$ & 0.232 & 0.83 & 0.3862 & 1.7796 & 0.631 \\
\hline $40-49$ & 22 & $6(27.3)$ & $16(72.7)$ & 0.802 & 0.64 & 0.2435 & 1.7003 & 0.371 \\
\hline $50-59$ & 28 & $17(60.7)$ & $11(39.3)$ & 8.193 & 3.09 & 1.3855 & 6.8953 & 0.004 \\
\hline $60-69$ & 40 & $10(25)$ & $30(75)$ & 2.481 & 0.54 & 0.2548 & 1.169 & 0.115 \\
\hline \multirow[t]{2}{*}{ 70-79 } & 30 & $6(20)$ & $24(80)$ & 3.763 & 0.40 & 0.1609 & 1.0342 & 0.052 \\
\hline & 280 & $101(36.1)$ & $179(63.9)$ & & & & & \\
\hline \multicolumn{9}{|l|}{ Sex } \\
\hline Male & 182 & $62(34)$ & $120(66)$ & 0.906 & 0.78 & 0.4705 & 1.2984 & 0.341 \\
\hline Female & 98 & 39 (39.8) & 59 (60.2) & & & & & \\
\hline \multicolumn{9}{|l|}{ Specimen } \\
\hline Pus & 120 & $44(36.7)$ & $76(63.3)$ & 0.032 & 1.04 & 0.6393 & 1.7119 & 0.857 \\
\hline Blood & 82 & $40(48.8)$ & $42(51.3)$ & 8.122 & 2.14 & 1.2618 & 3.6258 & 0.004 \\
\hline Throat & 46 & 8 (17.4) & 38 (82.6) & 8.329 & 0.32 & 0.1425 & 0.7147 & 0.004 \\
\hline Tissues & 20 & $4(20)$ & $16(80)$ & 2.412 & 0.42 & 0.1365 & 1.2929 & 0.120 \\
\hline Fluids & 12 & $5(41.7)$ & $7(58.3)$ & 0.170 & 1.27 & 0.3954 & 4.142 & 0.680 \\
\hline \multicolumn{9}{|l|}{ Type } \\
\hline Community acquired & 50 & $21(42)$ & $29(58)$ & 0.93 & 1.36 & 0.7277 & 2.5334 & 0.330 \\
\hline Hospital acquired & 230 & $80(34.8)$ & $150(65.2)$ & & & & & \\
\hline
\end{tabular}

$N$ total numbers of $S$. aureus isolations; $X^{2}$ Chi square; OR Odd ratio

$\mathrm{p}$ value $<0.05$ is considered as statistically significant 
Table 2 Antibiotics resistance pattern of MRSA and MSSA isolated from Peshawar region of Khyber Pakhtunkhwa, Pakistan

\begin{tabular}{|c|c|c|c|c|}
\hline Antimicrobial drugs & Drug susceptibility & MRSA n (\%) & MSSA n (\%) & $p$ value \\
\hline \multirow[t]{2}{*}{ Penicillin $(10 \mu \mathrm{g})$} & Susceptible & $0(0)$ & $0(0)$ & 1 \\
\hline & Resistant & $101(100)$ & $179(100)$ & \\
\hline \multirow[t]{2}{*}{ Amikacin $(30 \mu g)$} & Susceptible & $52(51.5)$ & $179(100)$ & $<0.0001$ \\
\hline & Resistant & $49(48.5)$ & $0(0)$ & \\
\hline \multirow[t]{2}{*}{ Chloramphenicol (30 $\mu \mathrm{g})$} & Susceptible & $78(77.2)$ & $179(100)$ & $<0.0001$ \\
\hline & Resistant & $23(22.8)$ & $0(0)$ & \\
\hline \multirow[t]{2}{*}{ 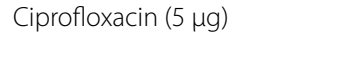 } & Susceptible & $20(19.8)$ & $137(76.5)$ & $<0.0001$ \\
\hline & Resistant & $81(80.2)$ & $42(23.7)$ & \\
\hline \multirow[t]{2}{*}{ Cefoxitin $(30 \mu \mathrm{g})$} & Susceptible & $0(0)$ & $179(100)$ & $<0.0001$ \\
\hline & Resistant & $101(100)$ & $0(0)$ & \\
\hline \multirow[t]{2}{*}{ Erythromycin $(15$ нg) } & Susceptible & $01(0.99)$ & $54(30.2)$ & $<0.0001$ \\
\hline & Resistant & $100(99.01)$ & $125(69.8)$ & \\
\hline \multirow[t]{2}{*}{ Gentamicin $(10 \mu \mathrm{g})$} & Susceptible & $44(43.6)$ & $167(93.3)$ & $<0.0001$ \\
\hline & Resistant & $57(56.4)$ & $12(6.7)$ & \\
\hline \multirow[t]{2}{*}{ Clindamycin $(2 \mu \mathrm{g})$} & Susceptible & $76(75.2)$ & $177(98.9)$ & $<0.0001$ \\
\hline & Resistant & $25(24.8)$ & $2(1.1)$ & \\
\hline \multirow[t]{2}{*}{ Minocycline $(30 \mu \mathrm{g})$} & Susceptible & $68(67.3)$ & $115(64.3)$ & 0.603 \\
\hline & Resistant & $33(32.7)$ & $64(35.7)$ & \\
\hline \multirow[t]{2}{*}{ Doxycycline $(30 \mu \mathrm{g})$} & Susceptible & $59(58.4)$ & $81(45.3)$ & 0.034 \\
\hline & Resistant & $42(41.6)$ & $98(54.7)$ & \\
\hline \multirow[t]{2}{*}{ Cotrimoxazole $(1.25 / 23.75 \mu \mathrm{g})$} & Susceptible & $67(65.3)$ & $167(93.3)$ & $<0.0001$ \\
\hline & Resistant & $34(33.7)$ & $12(6.7)$ & \\
\hline \multirow[t]{2}{*}{ Linezolid $(30 \mu \mathrm{g})$} & Susceptible & $101(100)$ & $179(100)$ & 1 \\
\hline & Resistant & $0(0)$ & $0(0)$ & \\
\hline \multirow[t]{2}{*}{ Fusidic acid $(10 \mu \mathrm{g})$} & Susceptible & $60(59.4)$ & $148(82.7)$ & $<0.0001$ \\
\hline & Resistant & $41(40.6)$ & $31(17.3)$ & \\
\hline \multirow[t]{2}{*}{ Rifampicin $(5 \mu \mathrm{g})$} & Susceptible & $82(81.2)$ & $179(100)$ & $<0.0001$ \\
\hline & Resistant & $19(18.8)$ & $0(0)$ & \\
\hline \multirow[t]{2}{*}{ Vancomycin $(30 \mu \mathrm{g})$} & Susceptible & $101(100)$ & $179(100)$ & 1 \\
\hline & Resistant & $0(0)$ & $0(0)$ & \\
\hline \multirow[t]{2}{*}{ Moxifloxacin $(5 \mu \mathrm{g})$} & Susceptible & $15(14.9)$ & $174(97.2)$ & $<0.0001$ \\
\hline & Resistant & $86(85.1)$ & $5(2.8)$ & \\
\hline
\end{tabular}

$p$ value $<0.05$ is considered as statistically significant

results revealed that $15.84 \%(\mathrm{n}=16)$ of MRSA isolates were positive for inducible clindamycin resistance.

MSSA showed high resistance (100\%) to penicillin followed by erythromycin $(69.8 \%)$ and doxycycline (54.7\%). In addition, MSSA showed $100 \%$ sensitivity to amikacin, chloramphenicol, cefoxitin, linezolid, vancomycin and rifampicin (Table 2).

\section{MIC for vancomycin}

As vancomycin is drug of choice against MRSA, therefore MIC of vancomycin was checked against MRSA isolates $(\mathrm{n}=79)$ which revealed that 42 isolates had MIC $2 \mu \mathrm{g} /$ $\mathrm{ml}$ followed by MIC $0.5 \mu \mathrm{g} / \mathrm{ml}(\mathrm{n}=12), 1 \mu \mathrm{g} / \mathrm{ml}(\mathrm{n}=12)$ $2.5 \mu \mathrm{g} / \mathrm{ml}(\mathrm{n}=6), 4 \mu \mathrm{g} / \mathrm{ml}(\mathrm{n}=6)$ and $8 \mu \mathrm{g} / \mathrm{ml}(\mathrm{n}=1)$.
Overall 7 isolates showed vancomycin MICs of 4 or $8 \mu \mathrm{g} /$ $\mathrm{ml}$ and thus were classified as VISA.

\section{Discussion}

Methicillin-resistant $S$. aureus has been reported as a major bacterial pathogen involved in both community and hospitalized infections worldwide due to its increased virulence and continuous growing antibiotics resistance (Chambers and DeLeo 2009). In the present study we found $36.1 \%$ frequency of MRSA among $S$. aureus isolates. A study conducted by Naeem et al. (2013) reported $31.5 \%$ frequency of MRSA in Peshawar. There is continuous increase in the global prevalence of MRSA but generally frequency of MRSA in European countries 
is lesser as compared to other parts of the world. The reason may be the implementation of strict antibiotic prescribing policies and infection control practices in European countries (Askari et al. 2012).

In the present study MRSA was most frequent in blood (48.7\%); similar finding was also observed earlier (Hassan et al. 2014). No statistically significant differences were found regarding frequency of MRSA in gender or group of infection origin (hospital acquired vs community acquired). MRSA infection was higher among the age group 50-59 years (60.71\%), which is in line with another study conducted in Pakistan which reported high prevalence of MRSA in age group +44 years (Khan et al. 2014).

The multi-drug resistance in MRSA isolates has been a major problem worldwide which leads to ineffective therapy and increase in treatment expenses (Köck et al. 2010). The present study highlighted that high number of MRSA were multi-drug resistant which is in line with the previous findings which reported high rate of multi-drug resistance in MRSA (Tiwari et al. 2008; Khan et al. 2013).

We have found that all MRSA isolates tested in the present study were fully sensitive to vancomycin so it may be used as the drug of choice for treating multidrug-resistant MRSA infections. Similar findings were reported by various previous studies (Singh et al. 2013; Sipahi et al. 2013; Bhatt et al. 2014; Chelliah et al. 2014). In addition, all isolates were sensitive to linezolid which is in line with other studies (Sipahi et al. 2013; Vijayamohan and Nair 2014). Linezolid has been approved by FDA for treating MRSA infection, so linezolid could be a possible alternative of vancomycin (Micek 2007). Previous study showed that patient treated with linezolid had significantly higher survival rate than those treated with vancomycin (Wunderink et al. 2003). All MRSA isolates were resistant to cefoxitin which is in line with other studies (Wang et al. 2013; Ahmad et al. 2015). MRSA resistance to quinolones (ciprofloxacin) was also found to be very high (80.19\%) in the current study. This correlates with an earlier finding where it has been shown that MRSA was $86.59 \%$ resistant to ciprofloxacin (Pandya et al. 2014).

The MIC profile revealed 7 (8.97\%) MRSA isolates as VISA. A previous study conducted at Pakistan also reported $13 \%$ frequency of VISA using MIC by E test (Hakim et al. 2007). In the current study, we observed $15.84 \%$ inducible clindamycin resistance in MRSA isolates which is in line with other studies (Yilmaz et al. 2007; Maninder et al. 2013). The high frequency of inducible clindamycin resistance in the present study and many other studies suggest that D-test should be adopted as routine practice in diagnostic microbiology to avoid clindamycin therapy failure (Maninder et al. 2013).

\section{Conclusion}

The present study highlighted high proportion of MRSA and their multidrug resistance in clinical settings at Peshawar. The MDR MRSA is a potential threat to the public health at Peshawar as this bug can disseminate in community causing severe clinical conditions. Vancomycin and linezolid could be the drugs of choice in treating MRSA infection. Furthermore, MIC of vancomycin should be performed routinely to screen out VISA. In depth studies are needed in different geographical areas of the Pakistan to better understand the epidemiology and molecular mechanisms of drug resistance in MRSA.

\section{Authors' contributions}

Study conception and design: MQ, HR, MH. Study collection and assembly of data: AU, AK. Study data analysis: JK, NM. Manuscript writing and final approval: AU, MQ. All authors read and approved the final manuscript.

\section{Author details}

${ }^{1}$ Department of Microbiology, Kohat University of Science and Technology, Kohat, Khyber Pakhtunkhwa 26000, Pakistan. ${ }^{2}$ Medical ICU Unit, Khyber Teaching Hospital, Peshawar, Khyber Pakhtunkhwa, Pakistan. ${ }^{3}$ Department of Biotechnology and Genetic Engineering, Kohat University of Science and Technology, Kohat, Khyber Pakhtunkhwa, Pakistan.

\section{Acknowledgements}

We are very thankful to Department of Pathology, Rehman Medical and Teaching Institute, Peshawar, Pakistan for the permission of samples collection and their expert technical assistance.

\section{Competing interests}

The authors declare that they have no competing interests.

Received: 8 November 2015 Accepted: 4 May 2016

Published online: 11 May 2016

\section{References}

Ahmad B, Khan F, Ahmed J, Cha SB, Shin M, Bashir S, Yoo HS (2015) Antibiotic resistance pattern and molecular epidemiology of methicillin-resistant Staphylococcus aureus colonization in burns unit of a tertiary care hospital in Peshawar, Pakistan. Trop J Pharm Res 13:2091-2099

Akinkunmi E, Lamikanra A (2012) A study of the intestinal carriage of antibiotic resistant Staphylococcus aureus by Nigerian children. Afr Health Sci 12:381-387

Anwar MS, Jaffery G, Bhatti KR, Tayib M, Bokhari SR (2004) Staphylococcus aureus and MRSA nasal carriage in general population. J Med Microbiol 14:661-664

Askari E, Soleymani F, Arianpoor A, Tabatabai SM, Amini A, Naderinasab M (2012) Epidemiology of mecA-methicillin resistant Staphylococcus aureus (MRSA) in Iran: a systematic review and meta-analysis. Iran J Basic Med Sci 15:1010-1019

Bhatt CP, Karki BMS, Baral B, Gautam S, Shah A, Chaudhary A (2014) Antibiotic susceptibility pattern of Staphylococcus aureus and methicillin-resistant Staphylococcus aureus in a tertiary care hospital. J Pathol Nepal 4:548-551

Chambers HF (2001) The changing epidemiology of Staphylococcus aureus. Emer Infect Dis 2:178-182

Chambers HF, DeLeo FR (2009) Waves of resistance: Staphylococcus aureus in the antibiotic era. Nat Rev Microbiol 7:629-641

Chelliah A, Thyagarajan R, Katragadda R, Leela KV, Babu RN (2014) Isolation of MRSA, ESBL and AmpC- $\beta$-lactamases from neonatal sepsis at a tertiary care hospital. J Clin Diagn Res 8:DC24

Clinical and Laboratory Standards Institute (2012) Performance standards for antimicrobial susceptibility testing, vol 32, no 3. CLSI M100-S22. Wayne, PA 
Deleo FR, Otto M, Kreiswirth BN, Chambers HF (2010) Community-associated meticillin-resistant Staphylococcus aureus. Lancet 375:1557-1568

Garner JS, Jarvis WR, Emori TG, Horan TC, Hughes JM (1988) CDC definitions for nosocomial infections, 1988. Am J Infect Control 16:128-140

Hakim ST, Arshed S, Iqbal M, Javaid SG (2007) Vancomycin sensitivity of Staphylococcus aureus isolates from hospital patients in Karachi. Pakistan. Libya J Med 2:176

Hassan AK, Mohammad M, Humera K, Samina N, Ahmed AK, Fridoon JA, Riffat M (2014) Prevalence, antibiotic susceptibility pat-tern and demographic factors related to methicillin resistant Staphylococcus aureus in Lahore, Pakistan. Int J Microbiol Adv Immunol 2:45-48

Holcomb HG, Durbin KJ, Cho M, Kyu J, Choi KJ, Darling ND, Allan D, Angerio AD (2008) Methicillin-resistant Staphylococcus aureus as a threat to public health: a cellular approach. Georgetown Univ J Health Sci 5:2

Khan SU, Mahmud MN, Chowdhury MA, Hakim MA (2013) Prevalence of multidrug resistant Staphylococcus aureus isolates in clinical specimens collected from local patients of Chittagong, Bangladesh. Chittagong Univ J Biol Sci 6:175-185

Khan S, Rasheed F, Zahra R (2014) Genetic polymorphism of agr locus and antibiotic resistance of Staphylococcus aureus at two hospitals in Pakistan. Pak J Med Sci. 30:172

Köck R, Becker K, Cookson B, van Gemert-Pijnen JE, Harbarth S, Kluytmans J (2010) Methicillin-resistant Staphylococcus aureus (MRSA): burden of disease and control challenges in Europe. Euro Surveill 15:1-9

Lee JH (2003) Methicillin (Oxacillin)-resistant Staphylococcus aureus strains isolated from major food animals and their potential transmission to humans. Appl Environ Microbiol 69:116489-116494

Maninder K, Gupte S, Aggarwal P, Manhas A, Bala M (2013) Prevalence and antimicrobial susceptibility pattern of methicillin resistant Staphylococcus aureus (MRSA) isolates in a tertiary care hospital in Punjab. J Evol Med Dent Scie 2:7841-7846

Micek ST (2007) Alternatives to vancomycin for the treatment of methicillinresistant Staphylococcus aureus infections. Clin Infect Dis 45:S184-S190

Naeem M, Adil M, Naz SM, Abbas SH, Khan I, Khan A, Khan MU (2013) Resistance and sensitivity pattern of Staphylococcus aureus: a study in lady reading hospital Peshawar. J Postgraduate Med Institute. 27:1

Oliveira DC, Milheirico C, Lencastre H (2006) Redefining a structural variant of Staphylococcal cassette chromosome mec, SCCmec type VI. Antimicrob Agents Chemother 50:3457-3459
Pandya N, Chaudhary A, Mehta S, Parmar R (2014) Characterization of methicillin resistant Staphylococcus aureus from various clinical samples at tertiary care hospital of rural Gujarat. J Res Med Dent Sci 2:49-53

Perwaiz S, Barakzi Q, Faroogi BJ, Khursheed N, Sabir N (2007) Antimicrobial susceptibility pattern of clinical isolates of methicillin-resistant Staphylococcus aureus. J Pak Med Assoc 57:2-4

Saleha A, Zunita Z (2010) Methicillin resistant Staphylococcus aureus (MRSA): an emerging veterinary and zoonotic pathogen of public health concern and some studies in Malaysia. J Anim Vet Adv 7:1094-1098

Salgado CD, Farr BM, Calfee DP (2003) Community-acquired methicillin-resistant Staphylococcus aureus: a meta-analysis of prevalence and risk factors. Clin Infect Dis 36:131-139

Singh U, Latha R, Setumadhavan K, Kavitha K, Gurumurthy MD (2013) Antimicrobial Profile of Methicillin Resistant Staphylococcus aureus (MRSA) Isolated from Skin \& Soft Tissue Infections (SSTI) from a Tertiary Care Hospital in Pondicherry. J Society Wound Care Res 6:30-35

Sipahi Resat O, Bardak-Ozcem S, Turhan T, Arda B, Ruksen M, Pullukcu H, Aydemir S (2013) Vancomycin versus linezolid in the treatment of methicillin-resistant Staphylococcus aureus meningitis. Surg infect 14:357-362

Tiwari HK, Sapkota D, Sen MR (2008) High prevalence of multidrug-resistant MRSA in a tertiary care hospital of northern India. Infect Drug Resist 1:57

Velasco D, del Mar Tomas M, Cartelle M, Beceiro A, Perez A, Molina F, Bou G (2005) Evaluation of different methods for detecting methicillin (oxacillin) resistance in Staphylococcus aureus. J Antimicrob Chemother 55:379-382

Vijayamohan N, Nair SP (2014) A study of the prevalence of methicillin-resistant Staphylococcus aureus in dermatology inpatients. Indian Dermatol Online J 5:441

Wang LX, Hu ZD, Hu YM, Tian B, Li J, Wang FX, Yang H, Xu HR, Li YC, Li J (2013) Molecular analysis and frequency of Staphylococcus aureus virulence genes isolated from bloodstream infections in a teaching hospital in Tianjin, China. Genet Mol Res 12:646-654

Wunderink RG, Rello J, Cammarata SK, Croos-Dabrera RV, Kollef MH (2003) Linezolid vs vancomycin: analysis of two double-blind studies of patients with methicillin-resistant Staphylococcus aureus nosocomial pneumonia. Chest 124:1789-1797

Yilmaz G, Aydin K, Iskender S, Caylan R, Koksal I (2007) Detection and prevalence of inducible clindamycin resistance in Staphylococci. J Med Microbiol 56:342-345

\section{Submit your manuscript to a SpringerOpen ${ }^{\circ}$ journal and benefit from:}

- Convenient online submission

- Rigorous peer review

Immediate publication on acceptance

- Open access: articles freely available online

- High visibility within the field

- Retaining the copyright to your article

Submit your next manuscript at $\boldsymbol{\nabla}$ springeropen.com 